\title{
Combined Experimental and Computational Investigation of the Fluorescence Quenching of Riboflavin by Cinnamic Alcohol Chemisorbed on Silica Nanoparticles
}

\author{
Valeria B. Arce, ${ }^{* \dagger, \ddagger}$ Juliana Scotto, ${ }^{\dagger}$ Patricia E. Allegretti, ${ }^{\S}$ Mauricio A. Melo Jr., " Claudio Airoldi," \\ María Laura Salum, ${ }^{\perp}$ Rosa Erra-Balsells, ${ }^{\perp}$ Reinaldo Pis Diez, ${ }^{\#}$ and Daniel O. Mártire* ${ }^{\dagger}$ \\ ${ }^{\dagger}$ Instituto de Investigaciones Fisicoquímicas Teóricas y Aplicadas, Universidad Nacional de La Plata, Casilla de Correo 16, Sucursal 4, \\ 1900 La Plata, Argentina \\ ${ }^{\ddagger}$ Centro de Investigaciones Ópticas, CONICET La Plata-CIC, CC 3, 1897 Gonnet, La Plata, Argentina \\ ${ }^{\S}$ LADECOR, Departamento de Química, Facultad de Ciencias Exactas, Universidad Nacional de La Plata, 1900 La Plata, Argentina \\ "Chemistry Institute, University of Campinas, P.O. Box 6154, 13084-971 Campinas, São Paulo, Brazil \\ ${ }^{\perp}$ CIHIDECAR-CONICET, Departamento de Química Orgánica, Facultad de Ciencias Exactas y Naturales, Universidad de Buenos \\ Aires, Pabellón II, 3 Piso, Ciudad Universitaria, 1428 Buenos Aires, Argentina \\ \#Centro de Química Inorgánica, Facultad de Ciencias Exactas, Universidad Nacional de la Plata, CC 962, 1900 La Plata, Argentina
}

Supporting Information

ABSTRACT: Riboflavin (vitamin B2) is usually present in water courses, lakes, and seas and acts as a photosensitizer in the photo-oxidation of a range of contaminants. However, little is known about the interaction of this compound with aromatics sorbed on silica sediments or on suspended silica particles. This article describes the modification and characterization of silica nanoparticles by condensation of the silanol groups of the particles with E-cinnamic alcohol. The reaction was confirmed by Fourier transform infrared spectroscopy (FTIR), solid-state ${ }^{13} \mathrm{C}$ and ${ }^{29} \mathrm{Si}$ crosspolarization magic angle spinning (CPMAS) NMR, reduction of the specific surface area measured by BET, thermal analysis, and fluorescence spectroscopy. Toxicity to the marine bacteria Vibrio fischeri of the modified particles was also measured. Riboflavin fluorescence was quenched in aqueous medium in the presence of dissolved E-cinnamic alcohol or in suspensions of the modified particles. The results are interpreted in terms of formation of 1:1 complexes between the ground states of riboflavin and the free or adsorbed cinnamic alcohol. Density functional theory (DFT) calculations in aqueous medium support the existence of the complex and explain the observed quenching of riboflavin fluorescence upon addition of cinnamic alcohol without affecting the emission maximum of riboflavin.

\section{INTRODUCTION}

The photochemistry and photophysics of isoalloxazines (10substituted 2,3,4,10-tetrahydro-benzo[g]pteridine-2,4-diones), and especially flavins (7,8-dimethyl substituted isoalloxazines), are of considerable interest due to the biological relevance of these compounds. It is widely recognized that the relatively strong fluorescence of flavins makes observation of their spectral and photophysical properties the best tool to evaluate the physical properties of binding sites of flavoproteins. ${ }^{1}$ The fluorescence of riboflavin (Rf) can be quenched by aromatics by different mechanisms, such as electron transfer, hydrogen bonding, hydrophobic effects, and $\pi-\pi$ interaction. ${ }^{2}$ Binding of riboflavin to hen egg riboflavin-binding protein results in an almost complete quenching of the riboflavin fluorescence, which is attributed to the ground-state $\pi$-stacking of the isoalloxazine ring and the aromatic residues. ${ }^{3} \pi$-Stacking of riboflavin with aromatic residues and the concomitant quenching of the riboflavin fluorescence has also been reported

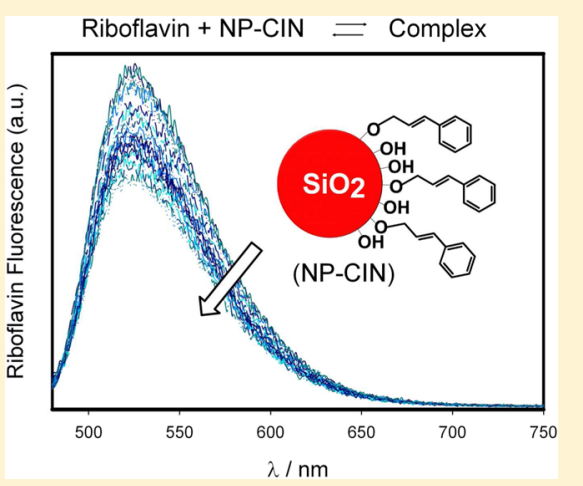

calculations in aquence un addition of cinnamic alcohol for other riboflavin-binding proteins. ${ }^{4}$ The remarkably low fluorescence yield of flavin adenine dinucleotide (FAD) with respect to free riboflavin was explained by the coplanar stacking of the flavin and adenine ring systems, most likely through a mechanism of photoinduced electron transfer of a nonfluorescent intramolecular ground-state complex between the isoalloxazine ring and the adenine moiety. ${ }^{5}$ Binding of $\mathrm{Rf}$ to salycilate ion in aqueous solution was also reported to yield a nonfluorescent complex. ${ }^{6}$

Besides the biological relevance of $\mathrm{Rf}$, this molecule is usually present in water courses, lakes, and seas $^{7}$ and acts as a photosensitizer in the photo-oxidation of a range of contaminants through both Type I and Type II processes. ${ }^{8-10}$ Chemical adsorption of organic compounds, such as alcohols

Received: May 19, 2014

Revised: June 19, 2014

Published: June 20, 2014 
and phenols, on the silica surface of sediments and on silicabased natural or manufactured nanomaterials in contaminated waters is a relevant process in the environment. ${ }^{11}$ For these reasons, we have recently investigated the interaction of the excited states of $\mathrm{Rf}$, a molecule employed as proxy of chromophore dissolved organic matter, ${ }^{12}$ with phenolic pesticides chemisorbed on silica nanoparticles. ${ }^{13,14}$

$E$-Cinnamic alcohol (E-3-phenyl-2-propene-1-ol, CIN) is an aromatic compound which belongs to the group of fragrances, i.e., chemicals incorporated in most cosmetic and other personal care products, such as sunscreens. ${ }^{15}$ Many cosmetic products, which are therefore released into domestic wastewater, also contain silica particles able to act as light scattering centers protecting the organic molecules from high load of UV energy. Recent studies have been focused on encapsulation or incorporation methods for photostable organic UV absorbers that reduce skin penetration ${ }^{16}$ and on the design of effective sunscreen carriers based on emerging technologies in controlled delivery. Among them, immobilization on silica particles of compounds structurally related to cinnamic alcohol was recently proposed. ${ }^{17}$

This background motivated us to investigate the interaction between $\mathrm{Rf}$ both with $\mathrm{CIN}$ and with silica nanoparticles modified with CIN (NP-CIN) in aqueous medium. To this purpose, fluorescence quenching experiments of $\mathrm{Rf}$ were performed. The results were complemented with $\mathrm{Rf}$ adsorption experiments on NP-CIN. Theoretical calculations within the context of the density functional theory (DFT) facilitated the interpretation of the experimental data.

The silica nanoparticles modification proceeded according to reaction 1 .
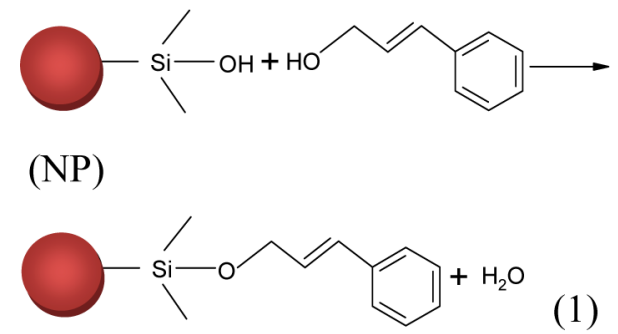

(NP-CIN)

The modified nanoparticles were characterized by Fourier transform infrared spectroscopy (FTIR) and ${ }^{13} \mathrm{C}$ and ${ }^{29} \mathrm{Si} \mathrm{NMR}$ spectra of the solid material and UV-vis absorption and fluorescence spectra and quantum yields of suspensions in different solvents. The specific surface area (SSA) was measured from nitrogen adsorption isotherms (BET method). The organic content of the modified particles was calculated from the mass loss of the samples in the thermogravimetry (TG) experiments. Additionally, the hydrolysis of the nanoparticles and their toxicity to the marine bacteria Vibrio fischeri were also studied.

\section{MATERIALS}

Fumed silica (Sigma; surface area, SSA $=390 \pm 40 \mathrm{~m}^{2} \mathrm{~g}^{-1}$; particle size estimated from the SSA $=0.007 \mu \mathrm{m})$ was employed. $o$-Xylene (Baker, analytical grade) was distilled onto molecular sieves previously dried at $250{ }^{\circ} \mathrm{C}$ for $4 \mathrm{~h}$.

Ethyl acetate (Ciccarelli, p.a.), $\mathrm{CaH}_{2}$ (Fluka), $\mathrm{CaCl}_{2}$ and $\mathrm{Rf}$ (Sigma-Aldrich), methanol, acetonitrile, and ethanol (Baker, analytical grade), dichloromethane and 2-propanol (Anedra), cinnamic alcohol (Meroar), $\mathrm{KOH}$ and $\mathrm{HCl}$ (Merck) were used without further purification. Distilled water $\left(>18 \mathrm{M} \Omega \mathrm{cm}^{-1}\right.$, $<20 \mathrm{ppb}$ of organic carbon) was obtained from a Millipore (Bedford, MA) system.

\section{E-Z PHOTOISOMERIZATION OF CINNAMIC ALCOHOL}

To determine the presence of $Z$ and/or $E$ isomers in the commercial cinnamic alcohol (CIN) employed for all the experiments, photoisomerization assays were performed. For this purpose, $5 \mathrm{~mL}$ solutions of CIN $(10 \mathrm{mg})$ in methanol (10 $\mathrm{mL}$ ) were irradiated with three lamps in a Pyrex container placed at $7.5 \mathrm{~cm}$ from the lamps, under magnetic stirring (lamp emission $\lambda=290-320 \mathrm{~nm}$, with emission maximum at $300 \mathrm{~nm}$; Rayonet, S. N. E. Ultraviolet Co., RPR-3000 Å lamp, Branford, CT 06405 U.S.A.). The ${ }^{1} \mathrm{H}$ NMR spectra in $\mathrm{Cl}_{3} \mathrm{CD}$ of the irradiated and nonirradiated samples recorded at $200 \mathrm{MHz}$ on a Bruker AC-200 spectrometer unambiguously show the existence of only the $E$ isomer in the nonirradiated sample (CIN) and the formation of the $Z$ isomer upon irradiation ( $E-$ $Z$ photoisomerization). ${ }^{18}$ See the Supporting Information, Figures S1 and S2.

\section{SYNTHESIS OF THE MODIFIED NANOPARTICLES}

The synthesis procedure was similar to those employed for obtaining silica nanoparticles modified with other organic groups. ${ }^{19,20}$ Briefly, $120 \mathrm{~g}$ of CIN was added to the mixture of 3 $\mathrm{g}$ of silica and $150 \mathrm{~mL}$ of $o$-xylene. The reaction was carried out in a Soxhlet extractor containing $1.5 \mathrm{~g}$ of $\mathrm{CaH}_{2}$ and a condenser with anhydrous $\mathrm{CaCl}_{2}$. The mixture was refluxed during $24 \mathrm{~h}$. The products were filtered through $20 \mathrm{~nm}$ nylon filters and washed with ethyl acetate. After drying the resulting gel at 0.1 Torr and at room temperature for $3 \mathrm{~h}$ and then at $120^{\circ} \mathrm{C}$ for 5 $\mathrm{h}$, a white powder was obtained. The modified particles (NPCIN) were characterized by UV-vis and FTIR spectroscopies, ${ }^{13} \mathrm{C}$ and ${ }^{29} \mathrm{Si}$ cross-polarization magic angle spinning (CPMAS) NMR, TG, and nitrogen adsorption isotherms. Characterization techniques and instrumentation, as well as the methodology employed for the hydrolysis and Vibrio fischeri assays, are described in the Supporting Information.

\section{ADSORPTION EXPERIMENTS}

Batch-mode adsorption kinetics experiments were carried out to investigate the contact time needed to reach equilibrium conditions. To this purpose, $2.0 \mathrm{mg}$ of $\mathrm{NP}-\mathrm{CIN}$ or NP was shaken with $5.0 \mathrm{~mL}$ of $\mathrm{Rf}$ aqueous solutions of $\mathrm{pH}=5.0$ in the concentration range of $5.6 \times 10^{-6}$ to $1.1 \times 10^{-4} \mathrm{M}$ in Erlenmeyer flasks at $25{ }^{\circ} \mathrm{C}$. The concentration of adsorbed $\mathrm{Rf}$ $\left([\mathrm{Rf}]_{\mathrm{ads}}\right)$ was calculated with eq I:

$$
[\mathrm{Rf}]_{\mathrm{ads}}=[\mathrm{Rf}]_{0}-[\mathrm{Rf}]_{t}
$$

where $[\mathrm{Rf}]_{0}$ and $[\mathrm{Rf}]_{t}$ are the molar concentrations of $\mathrm{Rf}$ at time $=0$ and time $=t$, respectively. To measure $[\mathrm{Rf}]_{t}$ the dispersions were filtered with $0.1 \mu \mathrm{m}$ filters. Both $[\mathrm{Rf}]_{0}$ and $[\mathrm{Rf}]_{t}$ were determined spectrophotometrically at $445 \mathrm{~nm}\left(\varepsilon^{445}\right.$ $\left.=10834 \mathrm{M}^{-1} \mathrm{~cm}^{-1}\right)$. The equilibrium condition was achieved after $2 \mathrm{~h}$ (results not shown). Thus, the equilibrium adsorption capacity of the adsorbents was obtained as $[\mathrm{Rf}]_{\mathrm{ads}, \mathrm{eq}} / C_{\mathrm{NP}}$ or $[\mathrm{Rf}]_{\mathrm{ads}, \mathrm{eq}} / C_{\mathrm{NP}-\mathrm{CIN}}$, where $[\mathrm{Rf}]_{\mathrm{ads}, \mathrm{eq}}$ is the value of $[\mathrm{Rf}]_{\mathrm{ads}}$ at equilibrium; $C_{\mathrm{NP}}$ and $C_{\mathrm{NP}-\mathrm{CIN}}$ are the concentrations of dispersed NP and NP-CIN (in $\mathrm{g} \mathrm{L}^{-1}$ ). The temperature was controlled at $298.0 \pm 0.1 \mathrm{~K}$ with a Grant model GD 1200 thermostat. 


\section{FLUORESCENCE SPECTROSCOPY}

Fluorescence measurements were made using a Jobin-Yvon SPEX Fluorolog spectrofluorometer (model FL3-11) equipped with a steady-state Xe lamp as the excitation source. The slit widths for both excitation and emission were fixed at $2 \mathrm{~nm}$. The band-pass gap was $1 \mathrm{~nm}$, and the integration time $0.3 \mathrm{~s}$. For recording the emission spectra, the excitation wavelength was set to $250 \mathrm{~nm}$. Fluorescence quantum yields of the solutions of $\mathrm{CIN}$ and suspensions of NP-CIN in different solvents were determined by comparison of the corrected fluorescence spectra of the samples with that of a fluorescence standard considering the refractive index correction. ${ }^{21}$ Tryptophan in water at $\mathrm{pH}=7.2$ was used as the standard $\left(\Phi_{\mathrm{F}}=0.14 \pm\right.$ $0.02) .^{22}$ The method assumes that the flux of absorbed photons is the same in the homogeneous and in microheterogeneous media. To guarantee this condition, for the NP the scattering component at the excitation wavelength was subtracted according to Ruiz et al. ${ }^{23}$ For the time-correlated single photon counting experiments, a Nanoled at $340 \mathrm{~nm}$ operating at 1 $\mathrm{MHz}$ was employed as the excitation source.

The steady-state fluorescence of $\mathrm{Rf}$ was measured at the excitation wavelength of $460 \mathrm{~nm}\left(A^{460}=0.1\right)$ with air-saturated solutions or suspensions preincubated under stirring (120 rpm) during $2 \mathrm{~h}$ in the temperature range of 283-303 K. For the time-correlated single photon counting experiments a Nanoled at $460 \mathrm{~nm}$ operating at $1 \mathrm{MHz}$ was employed as the excitation source.

\section{COMPUTATIONAL DETAILS}

One of the interests of the present work is the formation of a complex between Rf and CIN by means of $\pi-\pi$ interactions, hydrogen bonds, or a combination of those two types of interactions. Thus, ground-state geometry optimizations were performed within the context of the density functional theory $^{24-26}$ using the GGA exchange-correlation functional B97-D, which includes a long-range dispersion correction. ${ }^{27}$ The Hessian matrix of the energy with respect to the nuclear coordinates was diagonalized, and its eigenvalues were used to verify whether the optimized geometries were local minima or saddle points on the potential energy surface of the molecules and complexes.

Both geometry optimization of excited states and the calculation of electronic transitions were carried out within the framework of the time-dependent density functional theory. ${ }^{28,29}$ In this case, the hybrid GGA exchange-correlation functional PBE0 was used. ${ }^{30}$

Geometry optimizations were accomplished using the Def2SVP basis set for all the atoms. ${ }^{31}$ That basis set is of double- $\zeta$ quality augmented with polarization functions. For the calculation of the electronic transitions, the Def2-SVP basis set for nitrogen and oxygen was further augmented with diffuse functions. $^{32}$

Solvent effects (water) were included implicitly. The conductor-like polarizable continuum model ${ }^{33,34}$ was used to calculate the electrostatic contribution to the free energy of solvation, whereas the nonelectrostatic contributions were obtained with the SMD solvation model. ${ }^{35}$

Numerical integration of exchange-correlation functionals, both for geometry optimizations and electronic transitions, was performed on a grid containing 99 radial points and 302 angular points around each atom.
To compare with the experiments, simulated spectra were obtained by representing each electronic transition with Gaussian-shaped functions centered at the calculated band maximum. Finally, the whole spectrum was obtained by adding the contribution of every transition to the entire range of wavelengths. A Gaussian bandwidth of $0.25 \mathrm{eV}$ was used to interpret the experimental spectra.

All the calculations were carried out with the GAMESS-US package, ${ }^{36-38}$ and the figures were produced with the aid of the Gabedit program. ${ }^{39}$

\section{RESULTS AND DISCUSSION}

Characterization, Stability, and Toxicity of NP-CIN. FTIR, ${ }^{29} \mathrm{Si}$ NMR spectroscopic data of the solid NP-CIN samples, as well as UV-vis spectra of the water suspensions are in complete agreement with the modification of NP according to reaction $1 .{ }^{13} \mathrm{C}$ NMR are indicative of the presence of both chemically and physically sorbed CIN on the surface of the nanoparticles (see the Supporting Information).

The value of SSA obtained for the modified particles from BET experiments is $168 \pm 1 \mathrm{~m}^{2} \mathrm{~g}^{-1}$. The decrease in SSA compared to the value of the bare silica nanoparticles (in the present case $390 \mathrm{~m}^{2} \mathrm{~g}^{-1}$ ) upon silica functionalization with organic groups was observed for different systems. ${ }^{19,20}$

The percentage of organic groups in mass (\%OG = $7 \pm 1$ ) was calculated from the mass loss of the samples in the TG experiments from 200 to $700{ }^{\circ} \mathrm{C}$. ${ }^{40}$

The hydrolysis experiments show that after $2 \mathrm{~h}$ of boiling $2 \mathrm{~g}$ $\mathrm{L}^{-1}$ suspensions of $\mathrm{NP}-\mathrm{CIN}$ in acid $(0.1 \mathrm{M} \mathrm{HCl})$ or alkaline $(0.01 \mathrm{M} \mathrm{KOH})$ aqueous medium, the observed loss of surface organic matter is only $20 \%$ and $14 \%$, respectively. It was necessary to boil the suspensions of NP-CIN for $24 \mathrm{~h}$ to achieve mass losses of 50\% and $30 \%$ in acid and alkaline media, respectively. These data support the thermal stability of the samples during the fluorescence and adsorption experiments performed in the present work.

To complete the characterization of the new material NPCIN, toxicity measurements were performed. The toxicity of a chemical substance is characterized by the value of $\mathrm{EC}_{50}$, the concentration of the compound that produces an inhibition of bioluminescence of $50 \%$ compared to a blank assay. $V$. fischeri assays with NP showed no toxicity for suspensions of concentrations up to $1.0 \mathrm{~g} \mathrm{~L}^{-1}$. For aqueous suspensions of $\mathrm{NP}-\mathrm{CIN} \mathrm{EC}_{50}$ was found to be $25.4 \pm 0.8 \mathrm{mg} \mathrm{L}^{-1}$ (data not shown). Considering the \%OG value, this $\mathrm{EC}_{50}$ value can be expressed as $1.85 \pm 0.05 \mathrm{mg} \mathrm{L}^{-1}$ of bonded CIN. Comparative results with aqueous solutions of the free alcohol yield $\mathrm{EC}_{50}=$ $10.9 \pm 0.7 \mathrm{mg} \mathrm{L}^{-1}$. This means that a much higher toxicity is observed for the same amount of the alcohol when it is bonded to the nanoparticles compared to when it is free in solution. Similar results were found for other alcohols. ${ }^{20}$

For comparison of the toxicity of CIN and NP-CIN, we should consider that the toxicity of the nanoparticles is related to surface composition, which rules the toxicity of a nanomaterial. ${ }^{41}$ Consequently, the higher toxicity of the alcohol bonded to the nanoparticles could be caused by the favored bacterial adhesion to the hydrophobized surfaces. ${ }^{42}$ As a result, the local concentration of organic groups in contact with the bacteria is higher than in solution. ${ }^{42}$

Absorbance and Fluorescence of CIN in Solution and Suspensions of NP-CIN. Figure 1 shows the absorption spectrum of CIN in water. The absorption maxima in several 


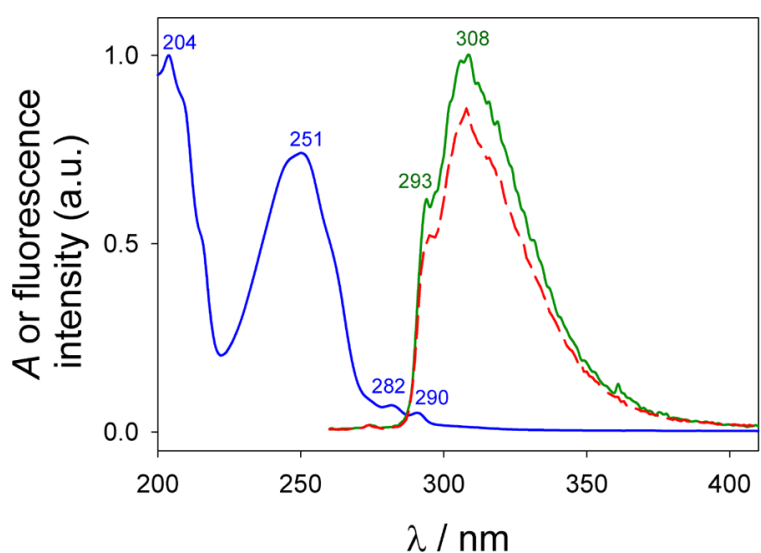

Figure 1. UV-vis spectrum and fluorescence emission spectrum of CIN (solid line) and fluorescence emission spectrum of NP-CIN (dashed line), in aqueous medium. The wavelengths ( $\mathrm{nm}$ ) of the main maxima and shoulders are shown.

solvents (all in the range from 250 to $252 \mathrm{~nm}$ ) are shown in Table $S 1$ in the Supporting Information.

The room-temperature emission spectra $\left(\lambda^{\text {exc }}=250 \mathrm{~nm}\right)$ of both CIN solutions and suspensions of NP-CIN in different solvents showed emission maxima in the range from 307 to 310 $\mathrm{nm}$ (see Supporting Information Table S1). The emission spectra of suspensions of the particles resulted very similar to those of solutions of CIN in the same solvent, as can be seen in Figure 1 for water.

As far as we know, the only reported emission spectrum of CIN in ethanol at $77 \mathrm{~K}^{43}$ exhibits a fingered pattern of five bands with emission maximum also at $308 \mathrm{~nm}$.

In order to assign the electronic transitions involved in the UV absorbance and fluorescence of CIN, DFT calculations were performed. The $E$ isomer of $\mathrm{CIN}$ was found to be $10.17 \mathrm{~kJ}$ $\mathrm{mol}^{-1}$ more stable than the $Z$ isomer, a fact that is in agreement with experimental evidence obtained for similar compounds. ${ }^{44}$

According to the Maxwell-Boltzman classical statistics, the $Z$ isomer should contribute less than $5 \%$ to the conformational space of CIN. The optimized geometry of E-CIN is shown in Figure 2. The $\mathrm{O}-\mathrm{C}-\mathrm{C}=\mathrm{C}$ torsion angle defining the relative orientation of the $\mathrm{OH}$ group with respect to the planar moiety

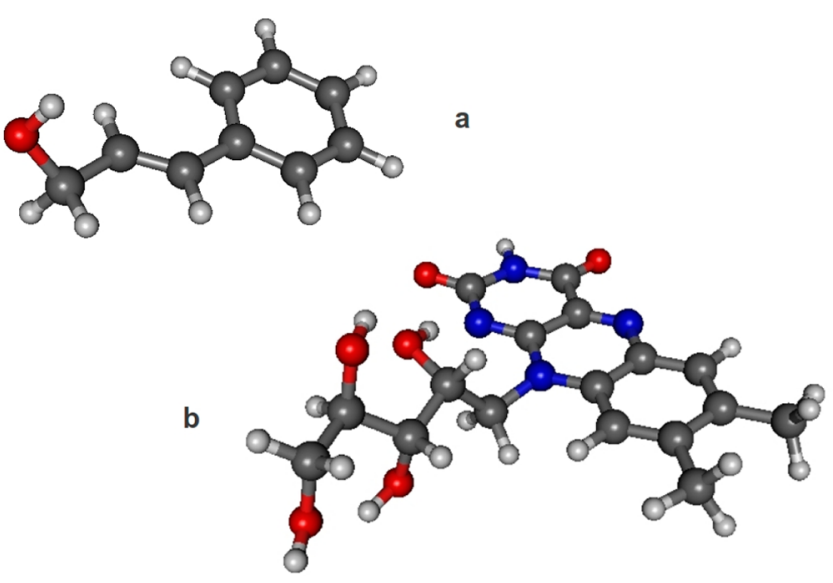

Figure 2. Optimized geometries of the $E$ isomer of cinnamic alcohol (a) and riboflavin (b) as obtained at the B97-D/Def2-SVP level of theory. is found to be $128.5^{\circ}$. For simplicity, we will refer to $E-C I N$ as CIN from now on.

The calculated absorption spectrum of CIN in water reveals three vertical transitions at 275,254 , and $220 \mathrm{~nm}$, in good agreement with the experimental data. Calculations indicate that the transition at $275 \mathrm{~nm}$ is dominated by an excitation from the highest occupied molecular orbital (HOMO) to the lowest unoccupied molecular orbital (LUMO), whereas the other two transitions exhibit many-body effects and are characterized by excitations from the HOMO -1 to the LUMO and from the HOMO to the LUMO + 1 with similar contributions. Figure S7 (Supporting Information) depicts the four relevant MOs needed to explain the absorption features of isolated $E$ form for CIN. The first excited state of CIN was optimized to study the $S_{1} \rightarrow S_{0}$ emission process. The transition from the LUMO to the HOMO during emission was calculated at $328 \mathrm{~nm}$.

Table S2 (Supporting Information) shows the measured fluorescence quantum yields $\left(\Phi_{\mathrm{F}}\right)$ for CIN and NP-CIN in different media. As can be seen in the table, the fluorescence quantum yields of the modified nanoparticles are, within the experimental error, coincident with or slightly lower than those measured for the alcohol in the same solvent. All the values are in the range of $0.07-0.13$. These results seem reasonable because there is no apparent reason (e.g., electronic interaction) to expect a lower fluorescence efficiency for the chemisorbed chromophore compared to that of the free alcohol.

Calculated Riboflavin Absorption and Fluorescence Spectra. The calculated absorption spectrum of $\mathrm{Rf}$ in water shows three vertical transitions at 422,359 , and $319 \mathrm{~nm}$. These results compare well with the vertical transition at $423 \mathrm{~nm}$ calculated elsewhere for the larger wavelength in dimethyl sulfoxide (DMSO) ${ }^{45}$ but differ from the experimental value of $468 \mathrm{~nm}$ obtained in ethanol at $77 \mathrm{~K}^{43}$ Calculations indicate that the transition at $422 \mathrm{~nm}$ corresponds to an excitation from the HOMO to the LUMO, whereas the other two transitions are dominated by excitations from the HOMO - 1 to the LUMO and from the HOMO -2 to the LUMO, respectively, suggesting that many-body effects are negligible. Figure S8 (Supporting Information) shows the four relevant MOs needed to describe the absorption spectrum of Rf. The first excited state of Rf was chosen to study the $S_{1} \rightarrow S_{0}$ emission process. The transition responsible for the emission was calculated at $483 \mathrm{~nm}$, in reasonable agreement with the vertical transition at $506 \mathrm{~nm}$ calculated elsewhere in DMSO. ${ }^{45}$ Those values differ, however, from the experimental emission line at $520 \mathrm{~nm}$ obtained here in water and also with data reported by Moore et $\mathrm{all}^{46}$

Riboflavin Fluorescence Quenching. The steady-state emission spectrum of $\mathrm{Rf}\left(\lambda^{\mathrm{exc}}=460 \mathrm{~nm}\right)$ in air-saturated suspensions of NP-CIN showed a decrease of the emission intensity $\left(I_{\mathrm{F}}\right)$ with increasing concentration of NP-CIN in the range from 0 to $1.6 \mathrm{~g} \mathrm{~L}^{-1}$ (Figure 3 ).

It is noteworthy that similar experiments with bare $\mathrm{SiO}_{2}$ nanoparticles in the same concentration range did not lead to fluorescence quenching of the Rf emission (data not shown). However, the emission of $\mathrm{Rf}$ was quenched upon addition of increasing amounts of CIN to the solutions. Thus, the quenching effect observed by the NP-CIN is due to the organic moieties (see below).

Conversely to the steady-state results, the singlet lifetime of $\mathrm{Rf}$ remained constant and equal to $4.7 \pm 0.2 \mathrm{~ns}$ after addition of 


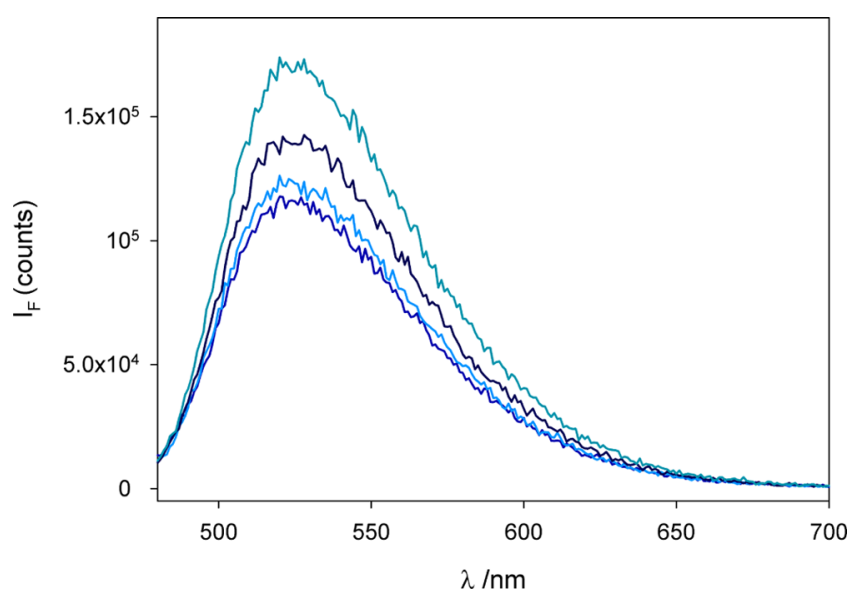

Figure 3. Riboflavin fluorescence intensity in the presence of variable amounts $\left(0,0.3,0.5\right.$, and $\left.0.9 \mathrm{~g} \mathrm{~L}^{-1}\right)$ of NP-CIN.

increasing amounts of CIN or NP-CIN. This fact pointed to the formation of ground-state complexes as the species responsible for the observed fluorescence quenching (static quenching). Assuming formation of 1:1 nonfluorescent groundstate complexes between $\mathrm{Rf}$ and $\mathrm{CIN}$, either free or chemisorbed on NP (NP-CIN), reactions 2 and 3 can be written.

$$
\begin{aligned}
& \mathrm{Rf}+\mathrm{CIN} \leftrightarrows \text { complex } \\
& \mathrm{Rf}+\mathrm{NP}-\mathrm{CIN} \leftrightarrows \mathrm{NP}-\text { complex }
\end{aligned}
$$

where NP-complex represents chemisorbed molecules of CIN:Rf complex. Data were fit to eq II.

$$
\frac{I_{0}}{I}=1+K[Q]
$$

where $I$ and $I_{0}$ stand for the Rf emission intensity in the presence and absence of the quencher, respectively. $K$ stands for $K_{2}$, the equilibrium constant of reaction 2 for the experiments performed in homogeneous medium, or for $K_{3}$, the equilibrium constant of reaction 3 for the experiments done with the NP-CIN suspensions.

From the slope of the linear plots of $I_{0} / I$ versus the molar concentration of cinnamic alcohol in solutions or in suspensions of NP-CIN (not shown), the equilibrium constant $K_{2}=130 \pm 3$ and $K_{3}=596 \pm 36$ are obtained at $293 \mathrm{~K}$. The van't Hoff plots of $\ln K_{2}$ and $\ln K_{3}$ versus $T^{-1}$ (Figure 4) yield the enthalpy changes $\Delta H^{\circ}{ }_{2}=-21 \pm 2$ and $\Delta H^{\circ}{ }_{3}=-31 \pm 6 \mathrm{~kJ}$ $\mathrm{mol}^{-1}$, respectively. These values are in excellent agreement with that measured for the formation of a 1:1 complex between $\mathrm{Rf}$ and the aromatic compound caffeine $(\Delta H=-24 \mathrm{~kJ}$ $\left.\mathrm{mol}^{-1}\right){ }^{47}$ The larger $\Delta H^{\circ}$ found for the NP-CIN system seems to suggest that $\mathrm{Rf}$ could present an additional interaction with the silica nanoparticle.

To further support the formation of a complex between $\mathrm{Rf}$ and $\mathrm{NP}-\mathrm{CIN}$, adsorption experiments of $\mathrm{Rf}$ on NP-CIN and bare $\mathrm{SiO}_{2}$ nanoparticles (NP) were performed. The linear plots of $[\mathrm{Rf}]_{\mathrm{ads}, \mathrm{eq}} / C_{\mathrm{NP}}$ versus $[\mathrm{Rf}]_{\mathrm{eq}}$ and $[\mathrm{Rf}]_{\mathrm{ads}, \mathrm{eq}} / C_{\mathrm{NP}-\mathrm{CIN}}$ versus $[\mathrm{Rf}]_{\text {eq }}$ (see Figure S9 in the Supporting Information) yielded $q_{\mathrm{e}}$ values of $2.2 \pm 0.3$ and $0.16 \pm 0.03 \mathrm{~g}^{-1} \mathrm{~L}$, respectively. These results are indicative of the preferential adsorption of $\mathrm{Rf}$ on $\mathrm{NP}-\mathrm{CIN}$ compared to NP and support the interaction between the ground-states of $\mathrm{Rf}$ and CIN.

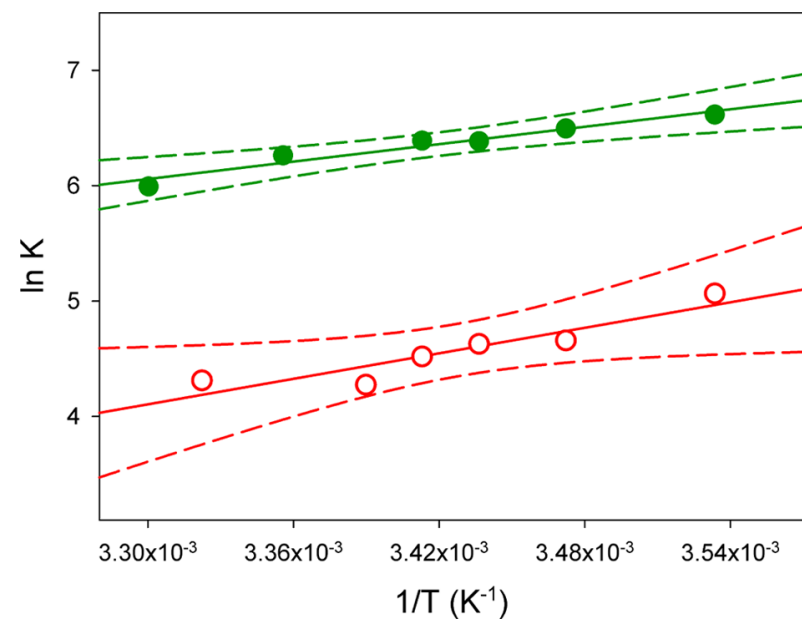

Figure 4. van't Hoff plots of $\ln K_{2}$ (open red circles) and $\ln K_{3}$ (closed green circles) vs $T^{-1}$.

To further support the experimental evidence for the formation of the CIN-Rf complex in water, a theoretical study was carried out. Four starting geometries differing mainly in the relative orientation of CIN with respect to $\mathrm{Rf}$ are constructed both for E-CIN-Rf and for Z-CIN-Rf. The comparison of the total electronic energies of the B97-D/Def2SVP optimized geometries shows that one stable complex formed by the $E$ conformer of CIN contributes to about $81 \%$ to the conformational space of CIN-Rf. On the other hand, the most stable Z-CIN-Rf complex contributes to about $11 \%$. To alleviate the effect of the incompleteness of the basis set, the basis set superposition error ${ }^{48}$ was calculated in the gas phase at the HF/Def2-SVP level of theory. The optimized geometries of the two more stable complexes are shown in Figure 5, in which it can also be seen that their BSSE-corrected complexation energies, calculated from the total free energies in solvent, are in the range from -64 to $-61 \mathrm{~kJ} \mathrm{~mol}^{-1}$. Those values greatly overestimate the enthalpy changes of -31 and $-21 \mathrm{~kJ} \mathrm{~mol}^{-1}$ reported in the present work. Interestingly, BSSE-corrected complexation energies of higher-energy conformations of the CIN-Rf complex are in the range from -51 to $-32 \mathrm{~kJ} \mathrm{~mol}^{-1}$, which are closer to experimental enthalpy values. The optimized geometries of the remaining complexes are shown in Figure S11 (see the Supporting Information). The optimized Cartesian coordinates of complexes I-VIII are shown in Tables S4-S11 (Supporting Information). A topological analysis carried out with Bader's quantum theory of atoms in molecules $^{49}$ on the equilibrium geometries of all the conformers indicates that $\mathrm{Rf}$ and CIN interact through hydrogen bonds involving the $\mathrm{OH}$ group of the $\mathrm{CIN}$ moiety and $\mathrm{O}$ or $\mathrm{N}$ atoms from $\mathrm{Rf}$, and through $\pi-\pi$ interactions between the CIN ring and the middle ring of Rf mainly. Nevertheless, an important difference in the nature of the intermolecular hydrogen bonds found between CIN and Rf lies in the fact that complexes I-VI form bonds characterized by the CIN$\mathrm{OH} \cdots \mathrm{Rf}$ interaction, whereas a $\mathrm{CIN}-(\mathrm{H}) \mathrm{O} \cdots \mathrm{Rf}$ bond is found in complexes VII and VIII; see Figure 5 and Supporting Information Figure S11 for the drawings and Table S3 (Supporting Information) for selected topological properties of the intermolecular hydrogen bonds. In light of these findings, we argue that complex $\mathbf{I}$ is formed when NPs are absent or when CIN is not bound to the NP. However, the thermal motion of water molecules reduces the $\pi-\pi$ interactions, and 

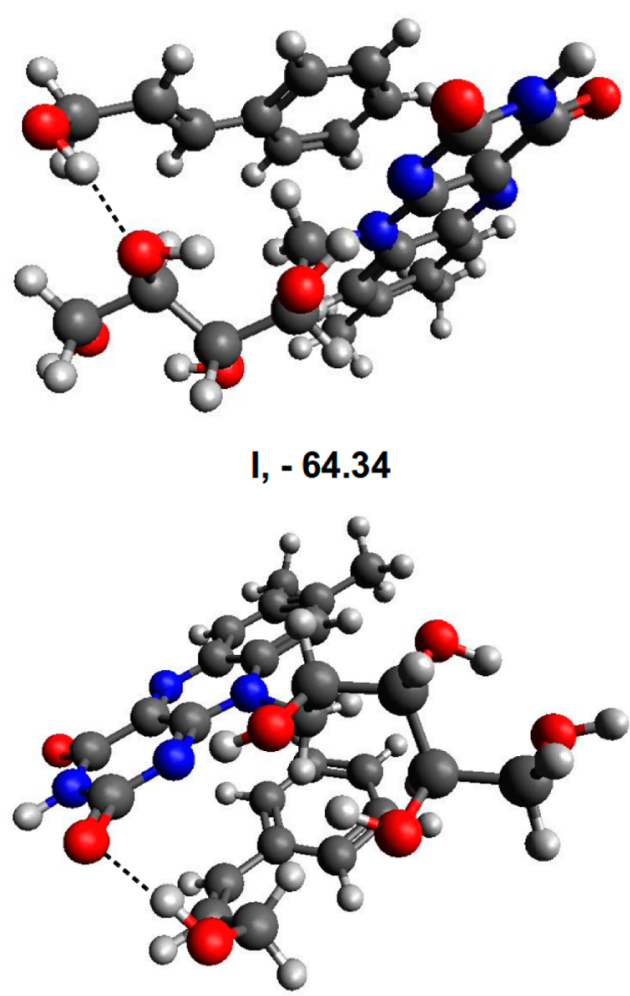

\section{II, - 60.96}

Figure 5. Optimized geometries of the most stable complexes formed by $E$ - and $Z$-cinnamic alcohol and riboflavin, I and II, respectively, as obtained at the B97-D/Def2-SVP level of theory. Complexation energies (in $\mathrm{kJ} / \mathrm{mol}$ ) are also given. Dashed lines show intermolecular hydrogen bonds.

consequently, the complexation energy of about $-60 \mathrm{~kJ} \mathrm{~mol}^{-1}$ becomes greatly reduced. When it is chemisorbed on the silica NPs, CIN looses the $\mathrm{H}$ atom of the $\mathrm{OH}$ group. Thus, complexes I-VI no longer exist and complexes VII and VIII are the new candidates to describe the CIN-Rf interaction. Moreover, due to the presence of the silica NP, the thermal motions are greatly diminished and the new complexation energy of about $-30 \mathrm{~kJ} \mathrm{~mol}^{-1}$ is almost unaltered.

The calculated absorption spectrum of complex I indicates that many-body effects are negligible for the CIN-Rf system and every transition is dominated by one excitation only. Thus, the four vertical transitions found at $486,442,372$, and $365 \mathrm{~nm}$ could be characterized by excitations from the HOMO to the LUMO, the HOMO - 1 to the LUMO, the HOMO - 2 to the LUMO, and the HOMO -3 to the LUMO, respectively. The calculated absorption spectra of complex $I$ is shown in Figure 6. Interestingly, complex II, the second most stable complex, and complex VII, one of the candidates to describe the CIN-Rf interaction after CIN chemisorption on the NP, exhibit four vertical transitions at 499, 429, 382, and $374 \mathrm{~nm}$, and at 501, 422, 401, and $353 \mathrm{~nm}$, respectively, which are characterized by the same excitations as the ones for complex $\mathbf{I}$.

The absorption spectra of an aqueous solution of $2.03 \times 10^{-5}$ $\mathrm{M} \mathrm{Rf}$ and an aqueous mixture containing $2.03 \times 10^{-5} \mathrm{M} \mathrm{Rf}$ and $1.49 \times 10^{-3} \mathrm{M}$ CIN are shown in the inset of Figure 6. From the value of $K_{2}$ it can be calculated that under these conditions $16 \%$ of the total amount of $\mathrm{Rf}$ is complexed, whereas $84 \%$ remains free. Figure 6 also shows the calculated absorption spectra of $\mathrm{Rf}$ and the above aqueous mixture. This latter

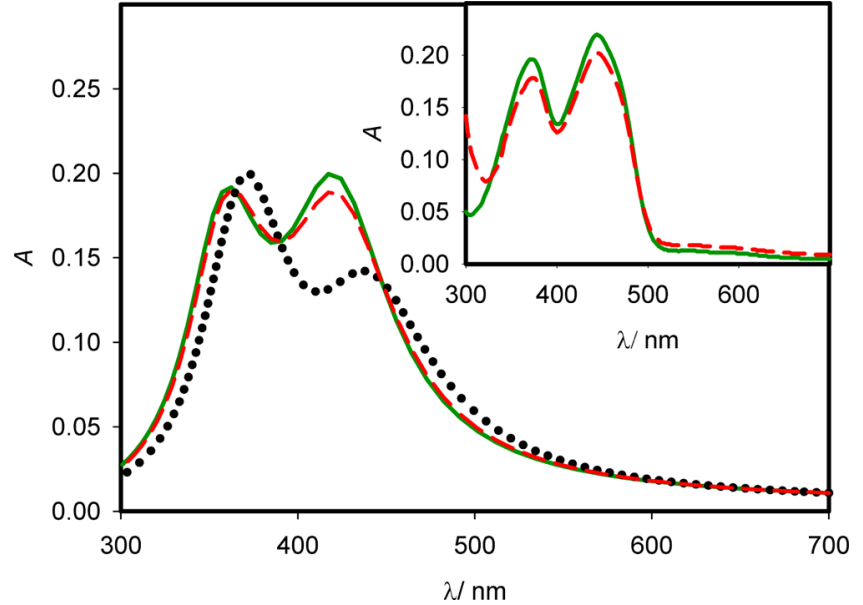

Figure 6. Calculated absorption spectra of $\mathrm{Rf}$ (solid line), complex I (dotted line), and a mixture containing $84 \%$ of $\mathrm{Rf}$ and $16 \%$ of complex I (dashed line). Inset: experimental absorption spectra of an aqueous solution of $2.03 \times 10^{-5} \mathrm{M} \mathrm{Rf}$ (solid line) and an aqueous mixture of $2.03 \times 10^{-5} \mathrm{M}$ Rf and $1.49 \times 10^{-3} \mathrm{M}$ CIN (dashed line).

spectrum was obtained as the sum of 0.16 times the calculated spectrum of complex I plus 0.84 times the calculated spectrum of Rf. Comparison of the experimental and theoretical spectra depicted in Figure 6 clearly shows that the spectral changes observed upon $\mathrm{Rf}$ complexation can be well-reproduced by the theoretical calculations.

Figure S10 (see the Supporting Information) shows the five relevant MOs needed to explain the absorption spectrum of the CIN-Rf complex I (see Supporting Information). It can be seen from the figure that the LUMO of complex I correlates with the LUMO of Rf, see Figure S8 (Supporting Information), whereas the HOMO and HOMO - 1 of the complex correlate with the HOMOs of CIN and Rf, respectively, see also Figure S7 (Supporting Information). The HOMO - 2 and HOMO 3 of the complex correlate with combinations of the HOMO 1 of $\mathrm{CIN}$ and the HOMO -2 of Rf. Thus, to investigate changes in the emission process of Rf after complexation with CIN, the geometry of the second excited state of complex I should be optimized. After geometry optimization of the second excited state of complex I, the LUMO $\rightarrow$ HOMO -1 vertical transition is found at $486 \mathrm{~nm}$.

Present results, then, indicate that the emission process of $\mathrm{Rf}$ is almost unaltered after complexation, passing from $483 \mathrm{~nm}$ in isolated Rf to $486 \mathrm{~nm}$ in complex I. Moreover, taking the oscillator strength as a measure of band intensities, calculations reveal that the band describing the emission process decreases its intensity from $0.3342 \mathrm{au}$ in isolated $\mathrm{Rf}$ to $0.2568 \mathrm{au}$ in complex I. These results support the interpretation trough the formation of $1: 1$ complexes (reactions 2 and 3) of both the observed quenching effect and constancy of the emission spectrum of Rf upon addition of CIN or NP-CIN, respectively.

For completeness, the geometry of the second excited state of complexes II and VII was also optimized. After optimization, the LUMO $\rightarrow$ HOMO - 1 vertical emission is found at 486 $\mathrm{nm}$ for both complexes, exactly the same value found for complex I. Moreover, the oscillator strength of the band described above is found to be 0.2379 and 0.2440 au for complexes II and VII, respectively, exhibiting a decrease with respect to the value of $0.3342 \mathrm{au}$ in isolated Rf. The fact that three distinct CIN-Rf complexes, I, II, and VII, with different equilibrium geometries, present very similar absorption and 
emission spectra reinforces the argument that $\mathrm{Rf}$ could bind the $\mathrm{NP}-\mathrm{CIN}$ system in a higher-energy conformation.

\section{CONCLUSIONS}

Silica nanoparticles modified with cinnamic alcohol (NP-CIN) were prepared and characterized. The alcohol fluorescence resulted unaffected by chemisorption on the particles. A higher toxicity of cinnamic alcohol to the marine bacteria $V$. fischeri was observed for the same amount of the alcohol when it is bonded to the nanoparticles compared to when it is free in solution.

Riboflavin fluorescence was quenched in aqueous medium in the presence of dissolved cinnamic alcohol or in suspensions of the NP-CIN. The results are interpreted in terms of formation of $1: 1$ complexes between the ground states of riboflavin and the free or adsorbed cinnamic alcohol. DFT calculations including solvent effects support the quenching results. Moreover, those calculations allow us to propose that CIN and $\mathrm{Rf}$ form different complexes when CIN is free and when CIN is chemisorbed on silica NPs.

\section{ASSOCIATED CONTENT}

\section{S Supporting Information}

${ }^{1} \mathrm{H}$ NMR spectra of irradiated and nonirradiated solutions of cinnamic alcohol, characterization of the modified nanoparticles (FTIR and UV absorption spectra, BET, thermogravimetry, ${ }^{13} \mathrm{C}$ and ${ }^{29} \mathrm{Si}$ NMR spectra), hydrolysis and toxicity assays, absorbance and fluorescence $\left(\lambda^{\mathrm{exc}}=250 \mathrm{~nm}\right)$ maxima obtained for solutions of cinnamic alcohol and suspensions of NP-CIN in different solvents, fluorescence quantum yields $\left(\Phi_{\mathrm{F}}\right)$ of CIN solutions and suspensions of NP-CIN in different media, relevant molecular orbitals to describe the absorption spectra of cinnamic alcohol, riboflavin, and the complexes, plots of $[\mathrm{Rf}]_{\mathrm{ads}, \mathrm{eq}} / C_{\mathrm{NP}}$ versus $[\mathrm{Rf}]_{\mathrm{eq}}$ and $[\mathrm{Rf}]_{\mathrm{ads}, \mathrm{eq}} / C_{\mathrm{NP}-\mathrm{CIN}}$ versus $[\mathrm{Rf}]_{\mathrm{eq}}$ for the adsorption of $\mathrm{Rf}$ on NP and NP-CIN, respectively, selected topological properties of the intermolecular hydrogen bonds found in complexes I-VIII, and optimized Cartesian coordinates of complexes I-VIII. This material is available free of charge via the Internet at http://pubs.acs.org.

\section{AUTHOR INFORMATION}

\section{Corresponding Authors}

*Phone: +54 221 4257430/7291. Fax: +54 221 4254642. Email: varce@quimica.unlp.edu.ar.

*Phone: +54 221 4257430/7291. Fax: +54 221 4254642. Email: dmartire@inifta.unlp.edu.ar.

\section{Notes}

The authors declare no competing financial interest.

\section{ACKNOWLEDGMENTS}

This work was supported by Grant PICT 2008 No. 0686 from Agencia Nacional de Promoción Científica y Tecnológica (ANPCyT, Argentina). M.L.S., R.E.-B., and R.P.D. are research members of CONICET. V.B.A. and D.O.M. are research members of Comisión de Investigaciones Científicas de la Provincia de Buenos Aires.

\section{REFERENCES}

(1) Sikorska, E.; Khmelinskii, I.; Komasa, A.; Koput, J.; Ferreira, L. F. V.; Herance, J. R.; Bourdelande, J. L.; Williams, S. L.; Worrall, D. R.; Insińska-Rak, M.; Sikorski, M. Spectroscopy and Photophysics of
Flavin Related Compounds: Riboflavin and Iso-(6,7)-Riboflavin. Chem. Phys. 2005, 314, 239-247.

(2) Datta, S.; Mukhopadhyay, C.; Bhattacharya, S.; Bose, S. K. Studies on the Riboflavin-Resorcinol Interaction in an Aqueous Medium and its pH Dependence. Spectrochim. Acta, Part A 2005, 62, $721-728$.

(3) Duurkens, R. H.; Tol, M. B.; Geertsma, E. R; Permentier, H. P.; Slotboom, D. J. Flavin Binding to the High Affinity Riboflavin Transporter RibU. J. Biol. Chem. 2007, 282, 10380-10386.

(4) Bieger, B.; Essen, L. O.; Oesterhelt, D. Crystal Structure of Halophilic Dodecin: A Novel, Dodecameric Flavin Binding Protein from Halobacterium salinarum. Structure (Cambridge, MA, U. S.) 2003, 11, 375-385.

(5) van den Berg, P. A. W.; Feenstra, K. A.; Mark, A. E.; Berendsen, H. J. C.; Visser, A. J. W. G. Dynamic Conformations of Flavin Adenine Dinucleotide: Simulated Molecular Dynamics of the Flavin Cofactor Related to the Time-Resolved Fluorescence Characteristics. J. Phys. Chem. B 2002, 106, 8858-8869.

(6) Datta, S.; Mukhopadhyay, C.; Bose, S. K. Molecular Complex Formation between Riboflavin and Salicylate in an Aqueous Medium. Bull. Chem. Soc. Jpn. 2003, 76, 1729-1734.

(7) Zeng, K.; Hwang, H.; Zhang, Y.; Yu, H. Identification of 6Aminochrysene Photoproducts and Study of the Effect of a Humic Acid and Riboflavin on its Photolysis. J. Photochem. Photobiol., B 2003, $72,95-100$.

(8) García, N. A.; Amat-Guerri, F. Photodegradation of Hydroxylated N-Heteroaromatic Derivatives in Natural-like Aquatic Environments. A Review of Kinetic Data of Pesticide Model Compounds. Chemosphere 2005, 59, 1067-1082.

(9) Escalada, J. P.; Pajares, A.; Gianotti, J.; Biasutti, A.; Criado, S.; Molina, P.; Massad, W.; Amat-Guerri, F.; García, N. A. Photosensitized Degradation in Water of the Phenolic Pesticides Bromoxynil and Dichlorophen in the Presence of Riboflavin, as a Model of their Natural Photodecomposition in the Environment. J. Hazard. Mater. 2011, 186, 466-472.

(10) Remucal, C. K.; McNeill, K. Photosensitized Amino Acid Degradation in the Presence of Riboflavin and its Derivatives. Environ. Sci. Technol. 2011, 45, 5230-5237.

(11) Wiesner, M. R.; Lowry, G. V.; Jones, K. L.; Hochella, M. F.; Di Giulio, R. T.; Casman, E.; Bernhardt, E. S. Decreasing Uncertainties in Assessing Environmental Exposure, Risk, and Ecological Implications of Nanomaterials. Environ. Sci. Technol. 2009, 43, 6458-6462.

(12) Vione, D.; Maddigapu, P. R.; De Laurentiis, E.; Minella, M.; Pazzi, M.; Maurino, V.; Minero, C.; Kouras, S.; Richard, C. Modelling the Photochemical Fate of Ibuprofen in Surface Waters. Water Res. 2011, 45, 6725-6736.

(13) Escalada, J. P.; Arce, V. B.; Porcal, G. V.; Biasutti, M. A.; Criado, S.; García, N. A.; Mártire, D. O. The effect of Dichlorophen Binding to Silica Nanoparticles on its Photosensitized Degradation in Water. Water Res. 2014, 50, 229-236.

(14) Escalada, J. P.; Arce, V. B.; Carlos, L.; Porcal, G. V.; Biasutti, M. A.; Criado, S.; García, N. A.; Mártire, D. O. Photodegradation Routes of the Herbicide Bromoxynil in Solution and Sorbed on Silica Nanoparticles. Environ. Sci.: Processes Impacts 2014, 16, 858-865.

(15) Becerril, E.; Lamas, J. P.; Sanchez-Prado, L.; Llompart, M.; Lores, M.; García-Jares, C. Analysis of Regulated Suspected Allergens in Waters. Talanta 2010, 83, 464-474.

(16) Li, C. C.; Chen, Y. T.; Lin, Y. T.; Sie, S. F.; Chen-Yang, Y. W. Mesoporous Silica Aerogel as a Drug Carrier for the Enhancement of the Sunscreen Ability of Benzophenone-3. Colloids Surf., B 2014, 115, 191-196.

(17) Noomane, A.; Hbaieb, S.; Bolzinger, M.; Briançon, S.; Chevalier, Y.; Kalfat, R. Effectiveness of Grafting Modes of Methoxycinnamate Sunscreen onto Silica Particles. Colloids Surf., A 2014, 441, 653-663.

(18) Mori, T.; Inoue, Y. In $C=C$ Photoinduced Isomerization Reactions; Griesbeck, A. G., Mattay, J., Eds.; Synthetic Organic Photochemistry; Marcel Dekker: New York, 2005; p 417.

(19) Arce, V. B.; Bertolotti, S. G.; Oliveira, F. J. V. E.; Airoldi, C.; Gonzalez, M. C.; Allegretti, P. E.; Mártire, D. O. Safranine-t Triplet- 
State Quenching by Modified Silica Nanoparticles. J. Phys. Chem. C 2011, 115, 18122-18130.

(20) Arce, V. B.; Bertolotti, S. G.; Oliveira, F. J. V. E.; Airoldi, C.; Arques, A.; Santos-Juanes, L.; Gonzalez, M. C.; Cobos, C. J.; Allegretti, P. E.; Mártire, D. O. Triplet State of 4-Methoxybenzyl Alcohol Chemisorbed on Silica Nanoparticles. Photochem. Photobiol. Sci. 2012, 11, 1032-1040.

(21) Valeur, B. Molecular Fluorescence. Principles and Applications; Wiley-VCH: Weinheim, Germany, 2002.

(22) Kirby, E. P.; Steiner, R. F. Influence of Solvent and Temperature upon the Fluorescence of Indole Derivatives. J. Phys. Chem. 1970, 74, 4480-4490.

(23) Ruiz, A. E.; Caregnato, P.; Arce, V. B.; Schiavoni, M. M.; Mora, V. C.; Gonzalez, M. C.; Allegretti, P. E.; Mártire, D. O. Synthesis and Characterization of Butoxylated Silica Nanoparticles. Reaction with Benzophenone Triplet-States. J. Phys. Chem. C 2007, 111, 7623-7628.

(24) Hohenberg, P.; Kohn, W. Inhomogeneous Electron Gas. Phys. Rev. 1964, 136, B864-B871.

(25) Kohn, W.; Sham, L. J. Self-Consistent Equations Including Exchange and Correlation Effects. Phys. Rev. 1965, 140, A1133A1138.

(26) Parr, R. G.; Yang, W. Density Functional Theory of Atoms and Molecules; University Press: Oxford, U.K., 1989.

(27) Grimme, S. Semiempirical GGA-Type Density Functional Constructed with a Long-Range Dispersion Correction. J. Comput. Chem. 2006, 27, 1787-1799.

(28) Dreuw, A.; Head-Gordon, M. Single-Reference Ab Initio Methods for the Calculation of Excited States of Large Molecules. Chem. Rev. 2005, 105, 4009-4037.

(29) Elliott, P.; Furche, F.; Burke, K. Excited States from TimeDependent Density Functional Theory. Rev. Comput. Chem. 2009, 26, 91-165.

(30) Adamo, C.; Barone, V. Toward Reliable Density Functional Methods without Adjustable Parameters: The PBE0 model. J. Chem. Phys. 1999, 110, 6158-6170.

(31) Weigend, F.; Ahlrichs, R. Balanced Basis Sets of Split Valence, Triple Zeta Valence and Quadruple Zeta Valence Quality for $\mathrm{H}$ to Rn: Design and Assessment of Accuracy. Phys. Chem. Chem. Phys. 2005, 7, 3297-3305.

(32) Rappoport, D.; Furche, F. Property-optimized Gaussian Basis Sets for Molecular Response Calculations. J. Chem. Phys. 2010, 133, 134105-134111.

(33) Barone, V.; Cossi, M. Quantum Calculation of Molecular Energies and Energy Gradients in Solution by a Conductor Solvent Model. J. Phys. Chem. A 1998, 102, 1995-2001.

(34) Cossi, M.; Rega, N.; Scalmani, G.; Barone, V. Energies, Structures, and Electronic Properties of Molecules in Solution with the C-PCM Solvation Model. J. Comput. Chem. 2003, 24, 669-681.

(35) Marenich, A. V.; Cramer, C. J.; Truhlar, D. G. Universal Solvation Model Based on Solute Electron Density and on a Continuum Model of the Solvent Defined by the Bulk Dielectric Constant and Atomic Surface Tensions. J. Phys. Chem. B 2009, 113, 6378-6396.

(36) GAMESS-US, version 11, August 2011 (R1).

(37) Schmidt, M. W.; Baldridge, K. K.; Boatz, J. A.; Elbert, S. T.; Gordon, M. S.; Jensen, J. H.; Koseki, S.; Matsunaga, N.; Nguyen, K. A.; $\mathrm{Su}$, S.; Windus, T. L.; Dupuis, M.; Montgomery, J. A. General Atomic and Molecular Electronic Structure System. J. Comput. Chem. 1993, 14, 1347-1363.

(38) Gordon, M. S.; Schmidt, M. W. In Theory and Applications of Computational Chemistry, the First Forty Years; Dykstra, C. E., Frenking, G., Kim, K. S., Scuseria, G. E., Eds.; Elsevier: Amsterdam, The Netherlands, 2005; pp 1167-1189.

(39) Allouche, A. R. Gabedit-A Graphical User Interface for Computational Chemistry Softwares. J. Comput. Chem. 2011, 32, 174182.

(40) de Farias, R. F.; Airoldi, C. Thermogravimetry as a Reliable Tool to Estimate the Density of Silanols on a Silica Gel Surface. J. Therm. Anal. 1998, 53, 751-756.
(41) Fubini, B.; Fenoglio, I.; Tomatis, M.; Turci, F. Effect of Chemical Composition and State of the Surface on the Toxic Response to High Aspect Ratio Nanomaterials. Nanomedicine 2011, 6, 899-920.

(42) Salerno, M. B.; Logan, B. E.; Velegol, D. Importance of Molecular Details in Predicting Bacterial Adhesion to Hydrophobic Surfaces. Langmuir 2004, 20, 10625-10629.

(43) Konschin, H.; Sundholm, F.; Sundholm, G. Fluorescence Characteristics of Lignin Model Compounds. Acta Chem. Scand., Ser. B 1976, 30, 262-266.

(44) Salum, M. L.; Robles, C. J.; Erra-Balsells, R. Photoisomerization of Ionic Liquids Ammonium Cinnamates: One-Pot Synthesis-Isolation of Z-Cinnamic Acids. Org. Lett. 2010, 12, 4808-4811.

(45) Klaumünzer, B.; Kröner, D.; Saalfrank, P. (TD-) DFT Calculation of Vibrational and Vibronic Spectra of Riboflavin in Solution. J. Phys. Chem. B 2010, 114, 10826-10834.

(46) Moore, W. M.; McDaniels, J. C.; Hen, J. A. The Photochemistry of Riboflavin VI. The Photophysical Properties of Isoalloxazines. Photochem. Photobiol. 1977, 25, 505-512.

(47) Gutman, D. E. Complex Formation Influence on Reaction Rate I. Effect of Caffeine on Riboflavin Base-Catalyzed Degradation Rate. J. Pharm. Sci. 1962, 51, 1162-1167.

(48) Boys, S. F.; Bernardi, F. Calculation of Small Molecular Interactions by Differences of Separate Total Energies-Some Procedures with Reduced Errors. Mol. Phys. 1970, 19, 553-566.

(49) Bader, R. F. W. Atoms in Molecules-A Quantum Theory; Oxford University Press: New York, 1990. 